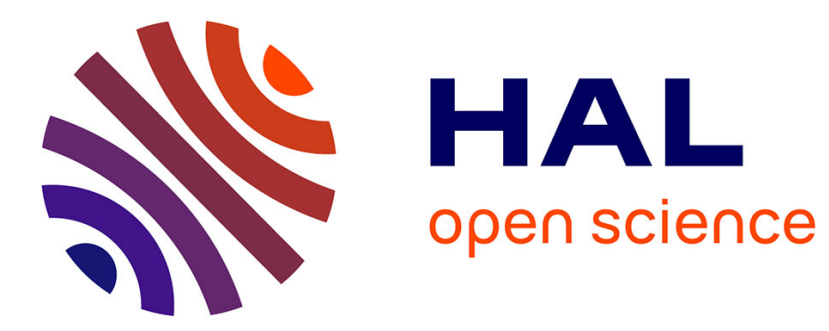

\title{
Does Dehazing Model Preserve Color Information?
}

\author{
Jessica El Khoury, Jean-Baptiste Thomas, Alamin Mansouri
}

\section{To cite this version:}

Jessica El Khoury, Jean-Baptiste Thomas, Alamin Mansouri. Does Dehazing Model Preserve Color Information?. SITIS 2014, Nov 2014, Marrakech, Morocco. 10.1109/SITIS.2014.78 . hal-01202981

\section{HAL Id: hal-01202981 \\ https://hal.science/hal-01202981}

Submitted on 22 Sep 2015

HAL is a multi-disciplinary open access archive for the deposit and dissemination of scientific research documents, whether they are published or not. The documents may come from teaching and research institutions in France or abroad, or from public or private research centers.
L'archive ouverte pluridisciplinaire HAL, est destinée au dépôt et à la diffusion de documents scientifiques de niveau recherche, publiés ou non, émanant des établissements d'enseignement et de recherche français ou étrangers, des laboratoires publics ou privés. 


\title{
Does Dehazing Model Preserve Color Information?
}

\author{
Jessica El Khoury, Jean-Baptiste Thomas, Alamin Mansouri \\ Le2i, Université de Bourgogne, \\ Bâtiment Mirande - UFR Sciences \& Techniques, \\ B.P. 47870 - 21078 Dijon Cedex, France \\ Email: jessica.el-khoury@u-bourgogne.fr
}

\begin{abstract}
Image dehazing aims at estimating the image information lost caused by the presence of fog, haze and smoke in the scene during acquisition. Degradation causes a loss in contrast and color information, thus enhancement becomes an inevitable task in imaging applications and consumer photography. Color information has been mostly evaluated perceptually along with quality, but no work addresses specifically this aspect. We demonstrate how dehazing model affects color information on simulated and real images. We use a convergence model from perception of transparency to simulate haze on images. We evaluate color loss in terms of angle of hue in IPT color space, saturation in CIE LUV color space and perceived color difference in CIE LAB color space. Results indicate that saturation is critically changed and hue is changed for achromatic colors and blue/yellow colors, where usual image processing space are not showing constant hue lines. we suggest that a correction model based on color transparency perception could help to retrieve color information as an additive layer on dehazing algorithms.
\end{abstract}

Index Terms - dehazing; perception; colorimetry; color fidelity; contrast enhancement; saturation; hue;

\section{INTRODUCTION}

Image enhancement becomes a highly recommended task in all imaging domains. For over a decade, researchers have been searching for an optimal method to get rid of degradation by light scattering along aerosols. A number of methods have been proposed and compared to each other. Moreover, each method is based on a specific hypothesis that may fail on some images, or when haze intensity increases.

We meet two types of dehazing methods, one with single input image and one with multiple input images. Researchers tried first to improve the variance between two different images of the same scene [6], [7]. Since then, they realized the utility to develop methods that restore images as well as extracting other quantities with minimal requirements of input data and user interaction: i.e. a single image. Thus, few methods have been developed. They are all based on the same model of haze. However, each one adopts a particular assumption. The results are more or less good depending on whether the processed image fits with the hypothesis or not. For each approach, modifications have been proposed to improve the performance in terms of restoration evaluation and computational time. The evaluation of restoration quality was limited to rate the number of recovered edges after processing, like by Hautiére et al. [12] or perceptual evaluation, like by Liu and Hardeberg [9]. These evaluation methods do not take into consideration the colorimetric aspect of restoration and they do not include it within the more general concept of perceptual quality as well.
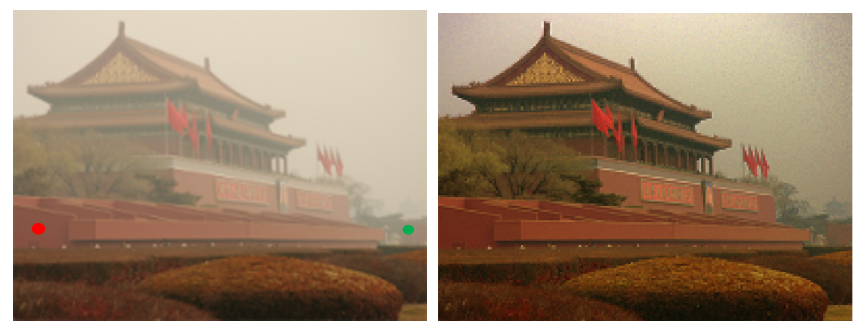

Fig. 1. Hazed input image and dehazed output image

Despite of the wide number of proposed approaches, the optimal result still far to be reached. Maintaining color fidelity remains a critical issue that was ignored in elder methods. Lately, researchers realized the importance to control its influence by associating non physical based methods to physical based methods because they assumed that the latter is not suitable for color correction [5]. Although color inaccuracy has been noticed, the problem has not been well quantified, neither solved. First methods focused on contrast/intensity rather than color fidelity.

Today, many application domains require to maintain color fidelity, where real color represents a fundamental property of objects as mentioned by Helmholtz."Colors have their greatest significance for us in so far as they are properties of bodies and can be used as marks of identification of bodies" [19]. Figure I shows a hazed image and the result we get when a dehazing method is applied. Dehazing enhances scene visibility by increasing contrast and saturating pixels. Does dehazing only saturate colors without affecting hue? How far color fidelity is maintained when haze increases? If the color is critically modified, how such shift could be adjusted, especially when original clear image is not available?

This paper addresses these questions by demonstrating how dehazing methods fail to preserve accurately original colors. Next sections are organized as follows. In section II, we review the existing works on image restoration and haze removal through common assumption and the most used model of color retrieval. In section III we point out the most important elements, which are potentially used to originate images and estimate their color alteration and we propose a method to test the fidelity of color recovering. In section IV we discuss how much color fidelity is maintained, and how far results do 
match color transparency expectations. Finally, we suggest that a correction based on color transparency perception models could be implemented.

\section{DEHAZING MODEL}

\section{A. State of the art}

Dehazing methods are divided into two categories: methods with single input image and methods with multiple input images. Methods belonging to the first category are currently more improved because they require less user interaction, and they do not need additional images captured under other conditions, which are often unavailable, to optimize rendering. Although, when we talk about methods with multiple images, a special equipment (like polarizers) is required, or some scenes under different weather conditions, or maybe various image types. These methods utilize dissimilarities between input images to boost haze-free image retrieval based on haze model.

In the following part, methods dealing with two input images are presented: Dehazing method using the dissimilarity between RGB and infrared images [7] [8], and the one using the sum of images with different polarizing angles [6]. Perhaps these methods are not suitable for many applications since they require more user interaction, but they could be more efficient in specific cases.

The first method is dealing with near-infrared light (NIR), which has stronger penetration capability than visible light due to its long wavelength. So, this light is less scattered by particles in the air. The advantage of deep penetration of NIR makes it possible to unveil the details, which could be completely lost in the visible range. The dissimilarity between RGB and NIR is exploited to estimate airlight characteristics. The problem of having a joint acquisition of visible and NIR light components in a single image was a problem up to today. However, we see emerging a new generation of sensors that permits the joint acquisition of visible and NIR light component in a single shot [23].

The second one employs different light polarizations. One of the causes of light polarization is the scattering. Scattered airlight intensity is divided into two components: $A^{\perp}$ and $A^{\|}$ that are perpendicular and parallel to the plan defined by the camera, the scatterer and the sun. Only when the light source is normal to the viewing direction, the airlight is totally polarized perpendicular to the plane of incidence. It can be eliminated if the image is captured through a polarizing filter oriented parallel to this plane. The polarization decreases as the direction of illumination deviates from 90 degrees and approaches to the viewing direction. Since that, the scattering of the directly transmitted light is unpolarized. Thus the polarization of the direct transmission is insignificant. In order to recover transmission, two images taken with different orientations of the polarizer have to be compared, and then airlight will be removed. This analysis is performed for each channel of RGB image.
Methods with single input image are gaining in interest, since they fit better automatic applications needs. Tan et al.[2] observe that haze-free images have larger local contrast and that the airlight is smooth. The corresponding results, after maximizing local contrast, tend to be oversaturated and can yield halo artifacts. The goal of this approach is not to fully recover the scene's original colors or albedo, it is just to enhance the contrast of the input image. Since the airlight, which is estimated by optimizing the data cost function is far from the actual value, the resulting images tend to have larger saturation values (of hue-saturation-intensity). This leads to unnatural restored images. Fattal [3] obtains, after assuming that the transmission and surface shading are uncorrelated, physically correct dehazed images, but his assumption might fail in cases of very dense haze. He et al. [1] introduce the simple and elegant dark channel prior, based on the observation that usually one channel by pixel is very dark in natural scenes. In other words, some pixels have very low intensity in at least one color (RGB) channel. The additive airlight, which increases with distance brightens these dark pixels. A depth map can thus be obtained and it is then used to recover the scene radiance.

Dark channel prior is the most popular approach. It provides comparable results with other approaches. It provides comparable results with other approaches, in terms of improving contrast, removing haze and maintaining natural colors. There are many attempts to optimize its performance (Like [16] and [17]).

Referred to [5], Zhang et al. tried to overcome color issue by grouping physics model based and non-physics model based methods (Retinex algorithm), which represent a subjective process that aims to improve the quality of the image according to the visual experience by enhancing the image contrast. This method adopts the same techniques of Dark Channel Prior, but when estimating transmission, non-physics model methods are introduced, namely, two bilateral filters to construct a new Retinex algorithm, which not only can enhance contrast and chroma, but also can reduce the halo phenomenon and noise. Retinex algorithm replaces the Dark Channel Prior to estimate the transmission, which is equivalent to the brightness image with less computational cost. Another way to separate image from haze veil is done by dividing image into illumination and reflectance by applying Retinex algorithm [4]. The haze veil is generated by computing the mean of illumination. It is then multiplied by the original image to get the depth map. Then luminance is transformed from RGB to the $\mathrm{YCbCr}$ color space, and the intensity component of haze veil is extracted to get the final haze veil. And then, the illumination is subtracted from the original image in the logarithmic domain. Finally, enhanced image seems dark, thus a post-processing of image enhancement is applied like dynamic range compression or histogram equalization. This process still reduces strongly the resolution in intensity. Tarel et al. [18] proposed a method characterized by its speed that allows it to be applied within real time processing applications. It consists on atmospheric veil inference by applying an original filter Median of Median 


\begin{tabular}{llll}
\hline Condition & Particle type & Radius $(\mu \mathrm{m})$ & Concentration $\left(\mathrm{cm}^{-3}\right)$ \\
\hline Air & Molecule & $10^{-4}$ & $10^{19}$ \\
Haze & Aerosol & $10^{-2}-1$ & $10^{3}-10$ \\
Fog & Water droplet & $1-10$ & $100-10$ \\
Cloud & Water droplet & $1-10$ & $300-10$ \\
Rain & Water drop & $10^{2}-10^{4}$ & $10^{-2}-10^{-5}$ \\
\hline
\end{tabular}

Fig. 2. Weather conditions and associated particles types, sizes and concentrations (adopted from McCartney (1975))

Along Lines, which preserves not only edges as median filter but corners as well (on gray level or RGB). Then a local smoothing is applied to soften the noise and artifacts. For an accurate visibility comparison between original and corrected images, a tone mapping is applied because corrected image is usually with a higher dynamic than the original one.

All of these methods are based on one haze model. Regardless of the adopted method, airlight and transmission are first estimated. Then, image radiance is deducted, thanks to haze model formula. Although, it is important to put the spotlight on the evaluation procedure, which compares methods regarding the computational time, the rate of new visible edges and the geometric mean ratios of visibility levels, and it uses as well the average gradient, which reflects the clarity of the image, the entropy that denotes the abundance of information included in the image, and the standard deviation, which represents the quality index that measures the contrast of the image.

\section{B. Definition}

Dehazing methods are developed in such a way to get rid of the veil and to enhance the global image quality. For that matter, physical based and non physical based approaches are joined to reach this aim. Physical based approaches handle issues from the physical aspect based on the hypothesis adopted to describe the original scene. These approaches achieve good results. However, they require additional assumptions of the scene like scene depth and multiple images. Non physical based approaches include image enhancing techniques such as the applying of bilateral and guided filter for image smoothing, histogram equalisation for contrast adjustment, etc. They suffer from less effectiveness on maintaining color fidelity.

Scattering that causes image disturbance, is mainly caused by a set of sparse atmospheric particles. The nature of scattering depends on the material properties, the shape and the size of particles. Thus, each weather condition scatters differently the emitted light. The exact form and intensity of scattering pattern varies dramatically with particle size.

All dehazing methods are dealing with haze and fog conditions. Haze is constituted of aerosol (small particles suspended in gas). Haze particles are larger than air molecules but smaller than fog droplets. It produces a distinctive gray or bluish hue and affects visibility. Fog has same origins as haze (volcanic ashes, foliage exudation, combustion products, sea salt) associated with an increase in relative humidity of an air. The size of water droplets is larger than the one of haze. It reduces visibility more than haze. Haze can turn into fog (transition state: mist).

For both conditions, haze and fog, it is Mie scattering, which is predominant. It is non-wavelength dependent. All wavelengths behave identically against scattering. Referring to Figure 2, the smallest radius haze particle is $10^{-2} \mu \mathrm{m}$. Assuming that 380 $\mathrm{nm}$ is the lowest visible wavelength. In order to apply Rayleigh scattering approach, particle size has to be up to about tenth of the wavelength of the light. A small part of haze particles satisfies this condition. Therefore, light wavelengths assumed to be similarly scattered according to Mie's theory.

A common model on which all hypothesis are based is the model of haze:

$$
I(x)=J(x) t(x)+A(1-t(x))
$$

$I(x)$ is the perceived intensity of the hazed image, $J(x)$ is the scene radiance of the original free-haze image and $t(x)=e^{-\beta z}$ is the direct transmission, which represents the non scattered light emanating from the object and is attenuated by the scattering along the line of sight. It describes the exponential attenuation of the scene radiance. $\beta$ is the scattering coefficient of the atmosphere and $z$ is the scene depth. The airlight corresponding to an object at an infinite distance is called atmospheric light $A_{\infty}$. Atmospheric light is always assumed to be isotropic. Airlight $A(1-t(x))$ is the light coming from an illuminant (sun) and scattered by the atmospheric particles towards the camera.

According to Mie scattering, which is non-wavelength dependent, all light wavelengths are identically scattered. Unlike underwater degradation, where colors go off successively throughout distance [10]. Therefore, haze model is not dependent on wavelength. It is only dependent on the distance between object and camera (represented by $z$ ), and the amount of haze covering the scene (represented by A). This means also, that there is no shift in hue of the original scene point color when passing through the haze. Therefore, we try to address this topic from the perceptual side. Even hue does not physically change while applying a scattering layer, perceptual hue could be differently interpreted.

According to MacAdam's paper [15], besides luminance contrast reduction, haze displaces chromaticities towards the white point. Consequently, it reduces the purity and the colorfulness of the scene. Because of chromatic adaptation, this effect is independent of the color of haze. It depends on the amount and depth of the haze. The reduction fraction of luminance contrast is approximately the same of purity reduction.

\section{EVALUATION OF COLOR SHIFT}

As we mentioned above, dehazing methods are all based on the same model, thus their impact on color would be the 
same. Therefore, we select only one to evaluate its performance. The popular dark channel prior approach is applied in order to calculate the elements we are searching for: airlight, transmission and image radiance. A colorimetric comparison study is conducted between original clear image and enhanced image. Some points located by different depths on hazed image are placed in adequate color spaces to identify the nature of color shifting.

\section{A. Indicators}

It is widely important to choose the adequate color space for a given processing and the suitable model to represent correspondent colors. Although the majority of dehazing methods use RGB color space, maybe the performance of dehazing will be better when using another color space. In this paper, we use CIE XYZ to embed haze via convergence model. We use CIE LAB to measure the perceptual color difference between hazed and dehazed color objects. CIE LUV is used to evaluate saturation evolution with dehazing process, and IPT color space to assess hue shift.

Although CIE XYZ is a metrological color space and CIE $\mathrm{LAB}$ is a color appearance space dedicated to the evaluation of small color differences. Similarly, CIE LUV is conceived for the same goal, but embeds an analytical expression of color saturation, which is very convenient here. This is due to the fact that while CIE LAB performs the chromatic adaptation by dividing by the illuminant, CIE LUV rather performs a subtraction of the illuminant. Both of these spaces have the major limit of curved constant hue lines, thus they are not suitable for the part of our analysis which considers hue. Therefore, we used the IPT color space for this aspect.

Many papers have cited that dehazing methods suffer from a common weakness: color fidelity deficiency [5], [4]. But this deficiency has never been clearly defined. This ambiguity pushes us to split up color components in order to precise how and how much each one is affected. The perceptually uniformed color space CIELUV clearly defines saturation [22]. This helps to point out how far saturation is affected with dehazing algorithms.

$$
s_{u, v}=13\left[\left(u^{\prime}-u_{n}^{\prime}\right)^{2}+\left(v^{\prime}-v_{n}^{\prime}\right)^{2}\right]^{1 / 2}
$$

$u^{\prime}$ and $v^{\prime}$ are the chrominance coordinates. $u_{n}^{\prime}$ and $v_{n}^{\prime}$ are the coordinates of the white point. The white point is the airlight color components. In synthetic image, airlight is the haze veil embed via convergence equation, and in real image it is the atmospheric light estimated by Dark Channel Prior (pixels with highest intensity of the hazed image among the top $0.1 \%$ brightest pixels in the dark channel).

The IPT space [20] was designed to be a simple approximation of color appearance specifically designed for image processing and gamut mapping. It is designed with fixing the hue nonlinearity of CIELAB. It consists on a linear transformations, along with some non linear processing. The second linear transformation goes from non linear cone sensitivities to an opponent color representation. Unlike other color spaces, such as CIEXYZ, IPT is characterized by having a very well aligned axis for constant hue [20]. It has a simple formulation and a hue-angle component with good prediction of constant perceived hue. I, P and T coordinates represent the lightness dimension, the red-green dimension and the yellow-blue dimension, respectively. Using a converting $3 \times 3$ matrix, when $\mathrm{I}, \mathrm{P}$ and $\mathrm{T}$ are computed from LMS, hue angle can than be computed through the inverse tangent of the ratio of $\mathrm{T}$ to $\mathrm{P}$ :

$$
h_{I P T}=\tan ^{-1}\left(\frac{T}{P}\right)
$$

\section{B. Color Transparency Model}

When a color object is viewed simultaneously partly directly and partly through a transparent filter but still perceived as the same surface, we talk about "color transparency". Translation and convergence in a linear trichromatic color space are supposed to lead to transparency perception. Humans are naturally able to separate chromatic properties of the transparent filter and the seen surface.

Referred to Metelli [14], with overlapping surfaces, three conditions are needed to perceive transparency: the uniformity of the transparent filter, the continuity of its boundaries and an adequate stratification.

Haze veil, which is spread along the scene can be considered as a non uniform transparent filter, because haze density depends on scene depth. Therefore, attenuation rate is controlled by: the scene depth and the haze intensity. Attenuation increases exponentially when the scene depth and/or the haze intensity increase $\left(t(x)=e^{-\beta z}\right)$. However, convergence model handles transparent filter without depth dimension.

According to D'Zmura et al. [11], translation and convergence in CIE xy lead to the perception of transparency. Color constancy revealed in presence of fog can be modelled by convergence model while taking into consideration shift in color and contrast. This was confirmed with asymmetric matching task [13].

Fog is simulated with convergence model as follows:

$$
b=(1-\alpha) a+\alpha f
$$

where $\mathrm{a}=\left(X_{a} Y_{a} Z_{a}\right)$ represents the tristimulus values of a surface, a convergence application leads to new tristimulus values $\mathrm{b}=\left(X_{b} Y_{b} Z_{b}\right) . \mathrm{f}=\left(X_{f} Y_{f} Z_{f}\right)$ is the target of convergence. $\alpha$ represents the amount of fog covering the surface: no fog if $(\alpha=0)$ and opaque fog if $(\alpha=1)$. Light that reaches the eye from the surface is the sum of: the original light emanating from the surface and the light that depends on the chromatic properties of the fog.

Fog differs from a transparent filter because chromatic effects of fog increase with depth, as the amount of fog intervening between surface and viewer increases. Unlike the transparent filter, fog imposes a chromatic transformation on underlying surfaces that depends strongly on the depth of a surface behind the filter. Referring to Hagedorn et. al [13], observers discount two aspects of the chromatic properties of fog: reduction in contrast and shift in the colors of lights from surfaces. Convergence model allows us to recover this shift. How does dehazing model meet this consideration?. As we mentioned 
above, not only haze intensity defined by $\alpha$ has to be taken into consideration, but also the scene depth. Thus, in this way the convergence model converges to the haze model.

\section{Simulation}

According to the convergence model, the simulation consists on embedding haze in CIE XYZ image. We applied the same model to RGB image in order to perform a cross validation with two different space basis. As it is shown in Figure 3, the original haze-free image is initiated as RGB and $\mathrm{XYZ}$ images. Haze was added to both images thanks to the convergence model with the same parameters values. Dark Channel Prior dehazing method is then applied to $\mathrm{RGB}_{R G B}^{H}$ and to $\mathrm{XYZ}_{R G B}^{H}$ hazed images, which are converted from $\mathrm{RGB}^{H}$. It is applied also to $\mathrm{XYZ}_{X Y Z}^{H}$ and to $\mathrm{RGB}_{X Y Z}^{H}$, which are converted from $\mathrm{XYZ}^{H}$. Four enhanced images are obtained for different values of $\alpha$. Three different values were assigned to $\alpha: 0.5,0.7$ and 0.9 . These values $\left(X_{f} Y_{f} Z_{f}\right)=$ (0.8 0.80 .8 ) were randomly assigned to haze layer along this simulation to represent a transparent gray veil. Although the same process may be used for a chromatic veil. Resulting images were converted to IPT images to evaluate hue changes by calculating the angle between the hue of the patch before and after dehazing processing, and to CIE LUV for saturation estimation. Comparison has been made between corrected images derived from the same original image type $\left(\left(\mathrm{RGB}_{X Y Z}^{D}\right.\right.$ and $\left.\mathrm{XYZ}_{X Y Z}^{D}\right),\left(\mathrm{RGB}_{R G B}^{D}\right.$ and $\left.\left.\mathrm{XYZ}_{R G B}^{D}\right)\right)$, and between the correspondent images derived from RGB and $\mathrm{XYZ}\left(\left(\mathrm{RGB}_{X Y Z}^{D}\right.\right.$ and $\left.\mathrm{RGB}_{R G B}^{D}\right),\left(\mathrm{XYZ}_{X Y Z}^{D}\right.$ and $\left.\left.\mathrm{XYZ} Z_{R G B}^{D}\right)\right)$. Curves that are shown in Figures 7 and 9, are resulting from hazed image where $\alpha=0.5$. The impact of haze intensity on saturation is shown in Figure 8. We used the Macbeth Color

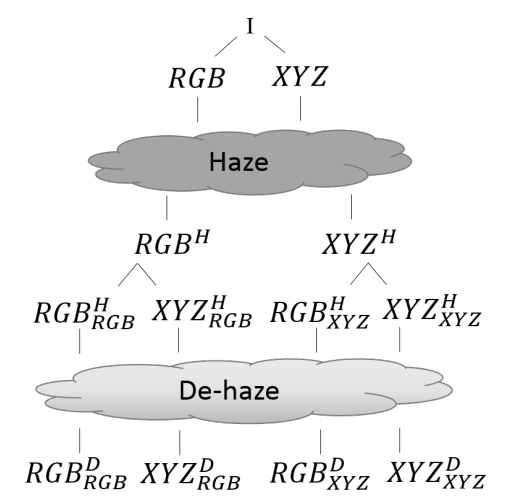

Fig. 3. Flowchart of the synthetic formation of analysed images

Checker [22] to simulate a flat object at a given distance from the camera. A synthetic fog image is composed of Macbeth Color Checker image and haze layer introduced by $\mathrm{f}$ in eq.4. Haze layer thickness is modified with the parameter $\alpha$. Distance and fog intensity are implicitly correlated: when fog intensity rises, it gives the same effect as if distance increases. Saturation and hue evolutions are calculated for each patch within three different values of $\alpha$. When $\alpha$ increases, it brings the apparent color toward veil color, such as far objects, which
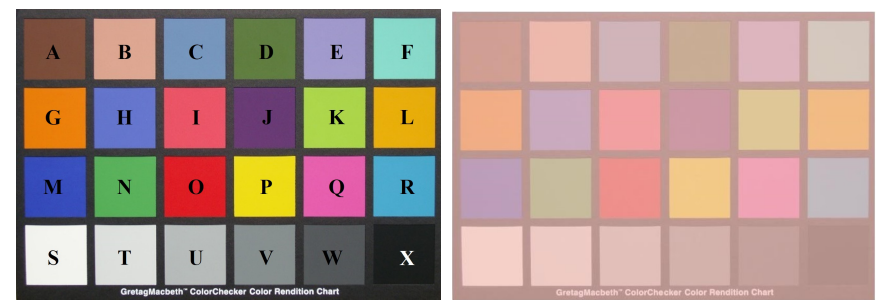

Fig. 4. Original and hazed images

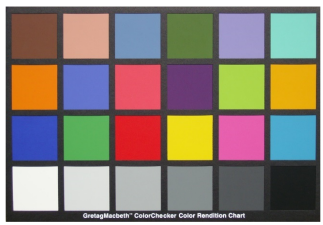

(a)

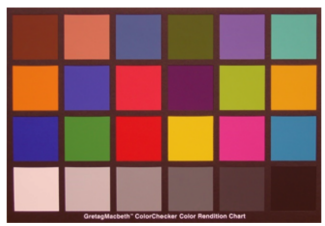

(c)

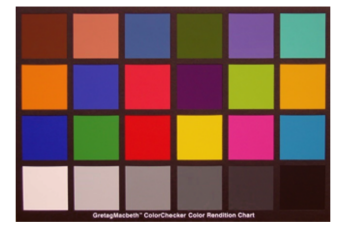

(b)

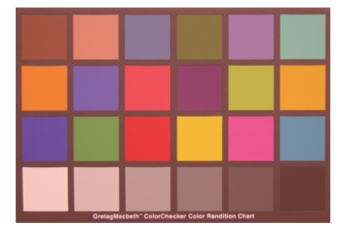

(d)

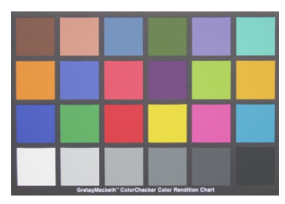

(e)

Fig. 5. Original RGB image and corrected images. (b): $\operatorname{RGB}_{X Y Z}^{D} \alpha=0.5$, (c): $\operatorname{RGB}_{X Y Z}^{D} \alpha=0.7$, (d): $\operatorname{RGB}_{X Y Z}^{D} \alpha=0.9$, (e): $\operatorname{RGB}_{R G B}^{D} \alpha=0.7$

are almost undistinguishable from haze.

On the other hand, saturation and hue are evaluated in a real image (see Figure I), where unlike synthetic image, transmission light emanating from far objects undergoes a severe attenuation. We choose two points, which are supposed to have the same initial color, located different depths and covered by non uniform haze veil.

\section{RESUlts}

Dehazing generally saturates pixels, whether it was applied to $\mathrm{XYZ}_{R G B}^{H}$ or $\mathrm{RGB}_{X Y Z}^{H}$. However, excluding black patch, achromatic patches $(\mathrm{S}, \mathrm{T}, \mathrm{U}, \mathrm{V}, \mathrm{W})$ are desaturated when original image is $\mathrm{XYZ}$ and they are slightly saturated when original image is RGB (Figure 7).

When RGB and XYZ are dehazed, if the original image is $\mathrm{XYZ}, \mathrm{RGB}_{X Y Z}^{D}$ will be more saturated (Figure 7(a)). On the other side, if the original image is $\mathrm{RGB}, \mathrm{XYZ}_{R G B}^{H}$ will be more saturated (Figure 7 (b)).

When the amount of haze increases, dehazing algorithms fail to retrieve accurately the original information. This reflects 

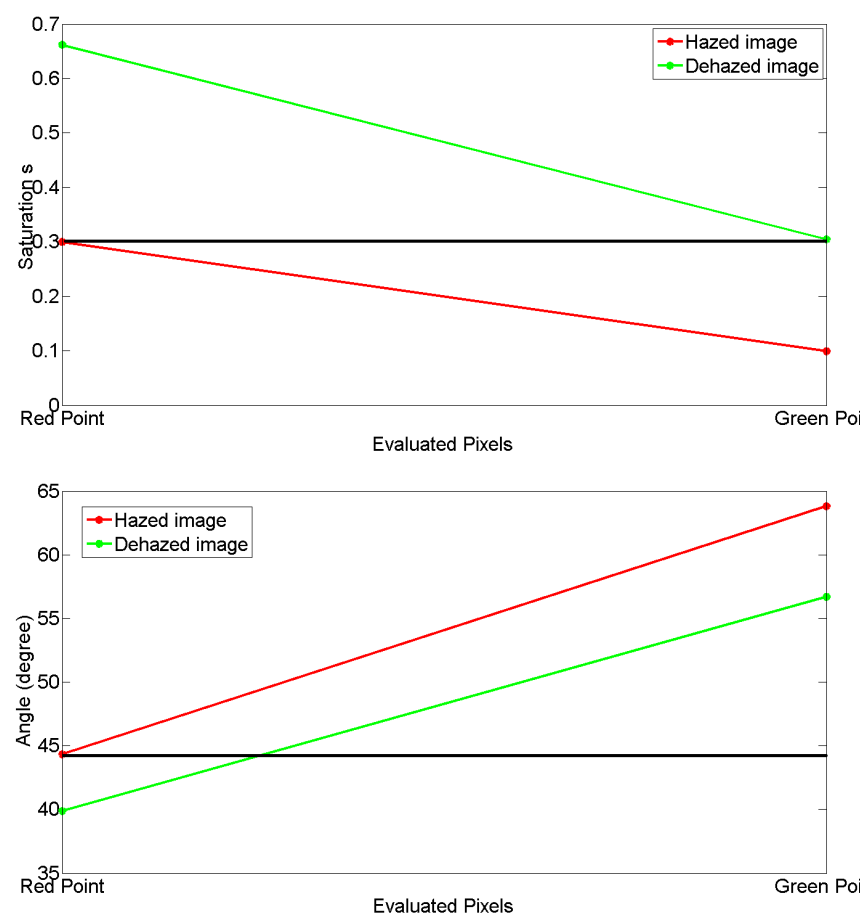

Fig. 6. Saturation and IPT angle difference of red and green dots in hazed and dehazed images

TABLE I

$\Delta E_{a b}^{*}$ BETWEEN RGB IMAGE AND RGB ${ }_{X Y Z}^{D}$

\begin{tabular}{|l|c|c|r|}
\hline Patch & $\alpha=0.5$ & $\alpha=0.7$ & $\alpha=0.9$ \\
\hline A & 22.7839 & 18.3615 & 8.9758 \\
B & 18.4932 & 17.3970 & 10.7179 \\
C & 17.2623 & 15.5641 & 12.8754 \\
D & 20.8679 & 17.0614 & 16.0229 \\
E & 21.3950 & 18.3785 & 11.6809 \\
F & 9.6771 & 8.2905 & 11.5722 \\
G & 5.8519 & 12.4668 & 30.2897 \\
H & 25.6762 & 20.1045 & 13.0253 \\
I & 24.0258 & 18.9867 & 4.7013 \\
J & 26.8635 & 19.3133 & 13.6619 \\
K & 17.7130 & 13.6940 & 15.0730 \\
L & 7.3845 & 14.2794 & 32.5890 \\
M & 26.7201 & 19.1785 & 17.8229 \\
N & 17.3591 & 13.7212 & 17.4900 \\
O & 14.6382 & 7.1959 & 16.7396 \\
P & 6.8475 & 10.3081 & 27.6221 \\
Q & 22.8840 & 17.8182 & 7.9751 \\
R & 9.3494 & 9.3503 & 15.0014 \\
S & 5.7544 & 7.9532 & 10.0632 \\
T & 7.9602 & 9.6492 & 10.6514 \\
U & 11.2635 & 12.0428 & 11.3961 \\
V & 14.3893 & 14.2373 & 12.2938 \\
W & 17.0809 & 16.3471 & 14.5248 \\
X & 21.9177 & 22.3346 & 26.4667 \\
\hline
\end{tabular}

a lesser capability to radically get rid of the veil and to consequently saturate objects color. Referring to Figure 8, when $\alpha$ increases, enhanced saturations decrease with a non proportional manner. Table I shows CIE76 $\left(\Delta E_{a b}^{*}\right)$ calculated for perceptual difference evaluation between original haze-free Macbeth Color Checker image and corrected image $\operatorname{RGB}_{X Y Z}^{D}$.

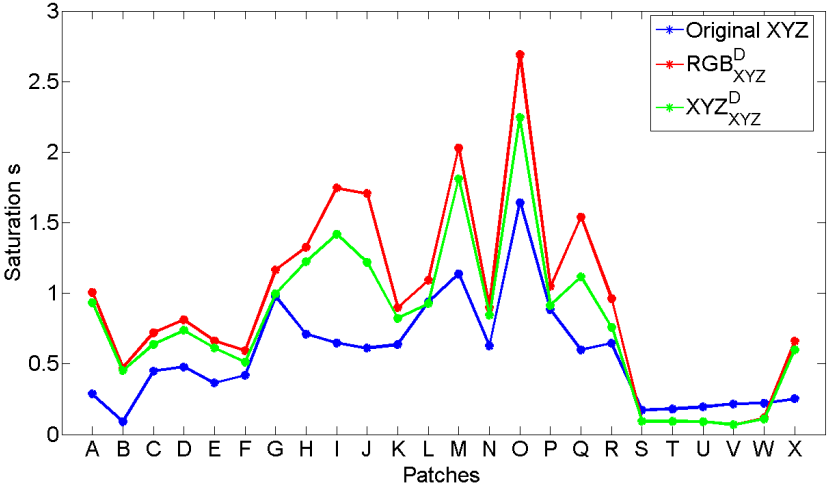

(a)

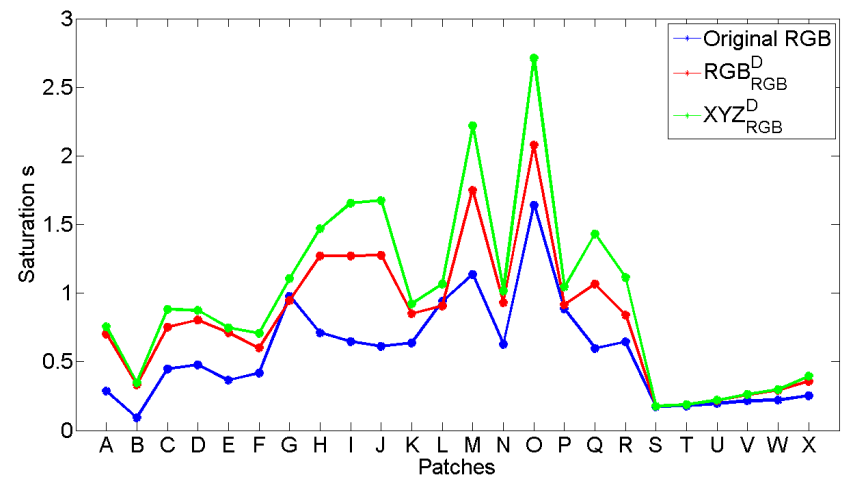

(b)

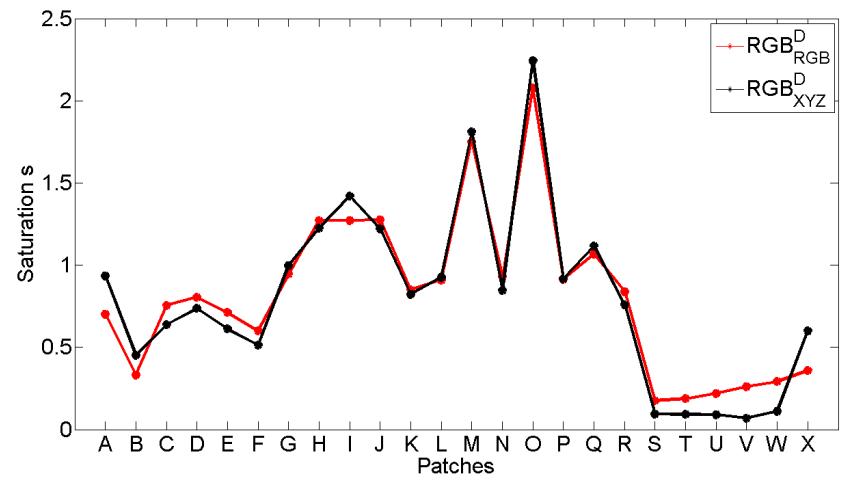

(c)

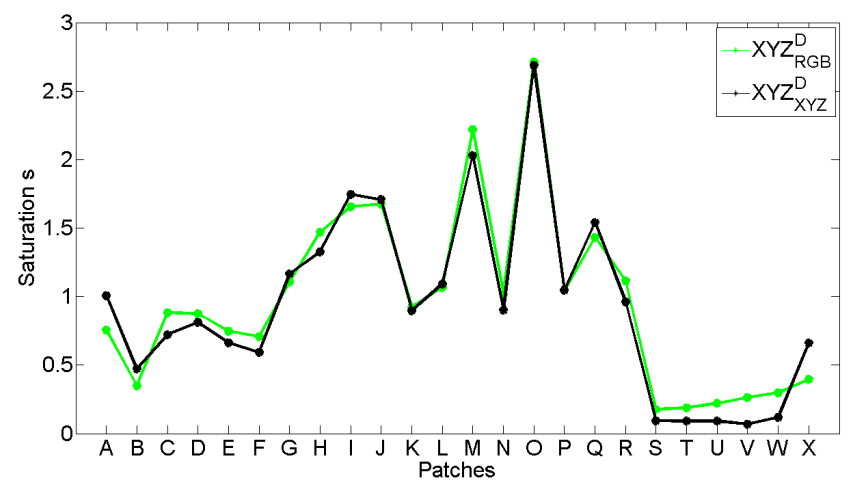

(d)

Fig. 7. Saturation evolution curves of rectified images in comparison with original clear images and other rectified images (normalized images) 


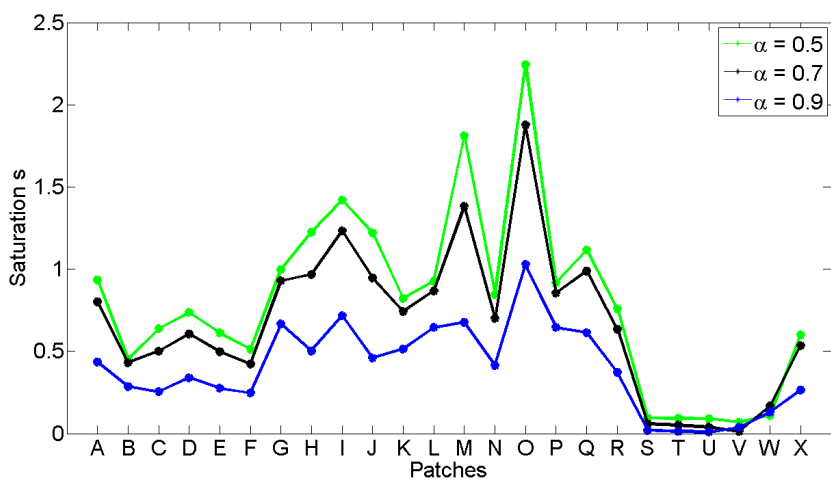

Fig. 8. Saturation evolution with $\alpha\left(\operatorname{RGB}_{X Y Z}^{D}\right.$ image)

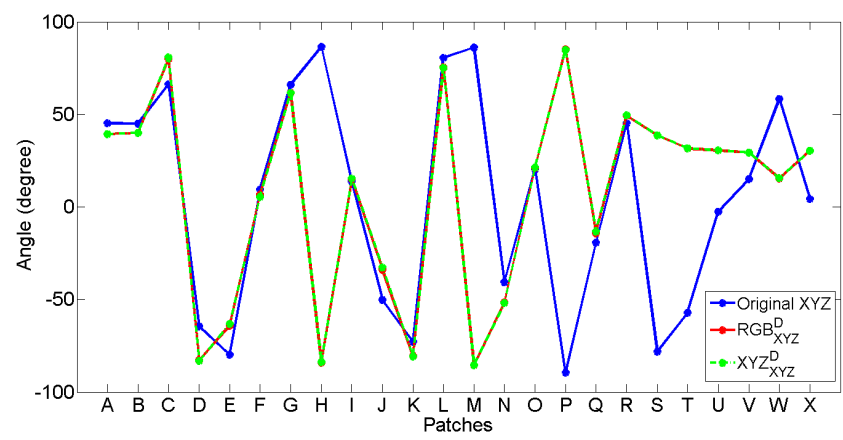

(a)

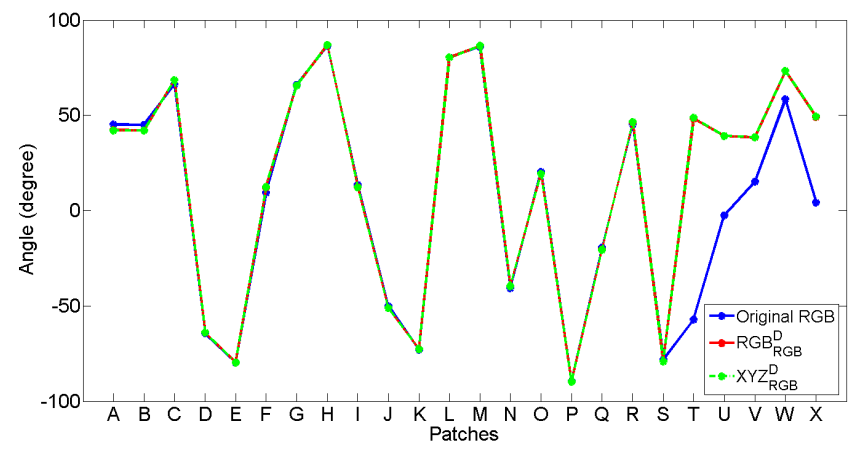

(b)

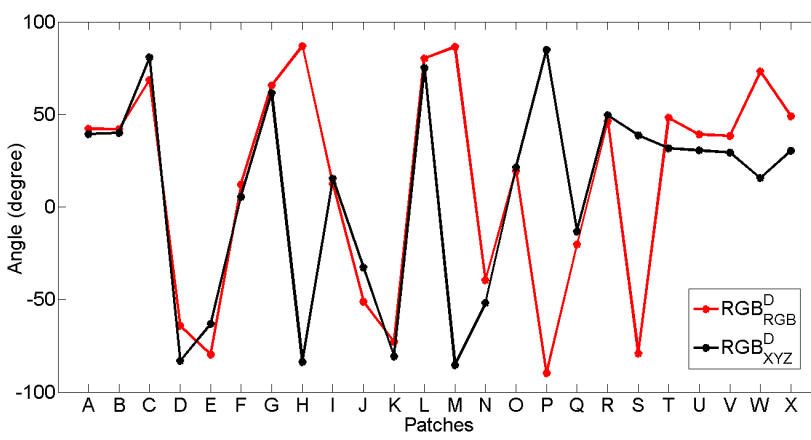

(c)

Fig. 9. Hue evolution curves of rectified images in comparison with original clear images and other rectified images (normalized images)
But these values would be much smaller when analyzing $\operatorname{RGB}_{R G B}^{D}$ (see Figure 5). These values evaluate the perceptual difference, which is noticed when we look at these images. Referring to Hagedorn et al. [13], the results of their experiment indicate that convergence fits the color matching data better with perceptual judgements in conditions with relatively more intervening fog. This can not be the case of photographed far object, because color information is partly lost along line of view. Thus, the difference between original haze free image and corrected image is important. Apparently, color rendering perception based on color convergence is not affected by haze intensity.

Unlike saturation, recovered hue of $\mathrm{RGB}_{X Y Z}^{D}$ and $\mathrm{RGB}_{R G B}^{D}$ fit the recovered hues of $X Y Z_{X Y Z}^{D}$ and $X Y Z_{R G B}^{D}$, respectively (see Figure 9 (a) and (b)). Thus, regardless color space, hue is identically recovered. But, they do not fit the hue of original color, especially when original image is XYZ. In this case, the correspondent achromatic and blue/yellow hues (patches $\mathrm{H}, \mathrm{M}$ and $\mathrm{P}$ ) before and after dehazing are not placed on a constant hue line. However, when original image is RGB, hue difference is important only on achromatic colors, except white (see Figure 9(b)).

The results of saturation and hue evolution of two indicated points of the real image (Figure I) shown in figure 6, indicate that they both vary when haze covers the image. Both saturation and hue of the green point converge toward the hue and the saturation of the red point before dehazing, which initially have the same color.

\section{CONCLUSION}

We proposed a simulation based process to evaluate how hue and saturation are affected by dehazing. Saturation was evaluated in CIE LUV color space, thanks to saturation formula. Hue was evaluated in IPT color space, which is characterized by the location of different points with reasonably good constant hue lines. Saturation and hue are both affected through free-haze image retrieval process. Colors are globally saturated when dehazing is applied. However, hue is not affected uniformly over different patches under the studied color spaces.

Thus, dehazing process achieves haze elimination, which is created by convergence model without considering attenuation caused by haze along the line of view. Color shift detected on hue and saturation could be evaluated and corrected in relation to attenuation coefficient and scene depth as well. Although this work narrowly examines dehazing model consequences, it brings on new questions.

The work described in this paper is only the beginning of a large project, where the impact of different color spaces of treated images will be studied, with different simulated illuminant conditions. Although hazing model in atmospheric environment is supposed to be a non spectral based, it seems significant to evaluate hue evolution when the initial information is spectral based.

As a continuity of this work, we suggest two methods to control color during the dehazing process: IPT space or any 
constant hue line space might be used as processing space in order to preserve hue angle. A mean has to be found to retrieve saturation, and may be based on a convergence model from perception of transparence. Saturation retrieval should take into considerations observer preferences. For artistic issue, it is suitable to increase saturation while maintaining natural colors. But in other cases, it is mandatory to accurately restore original saturation.

However, more visual aspects might be considered. Since the haze image is more likely to be less intense, while increasing the intensity we could see some adaptation effects such as Abney effect.This hue shift would not be solved by a space with constant hue lines.

\section{ACKNOWLEDGMENT}

The authors thanks the Open Food System project for funding. Open Food System is a research project supported by Vitagora, Cap Digital, Imaginove, Aquimer, Microtechnique and Agrimip, funded by the French State and the FrancheComt Region as part of The Investments for the Future Programme managed by Bpifrance, www.openfoodsystem.fr.

\section{REFERENCES}

[1] He, Kaiming, Jian Sun and Xiaoou Tang, "Single image haze removal using dark channel prior.", Computer Vision and Pattern Recognition, CVPR 2009. IEEE Conference on, pp. 1956-1963. IEEE, 2009.

[2] Tan, Robby T, "Visibility in bad weather from a single image IEEE Conference on Computer Vision and Pattern Recognition, CVPR, pp. 1-8, Year 2008

[3] R. Fattal, "Single image dehazing", International Conference on ComputerGraphics and Interactive Techniques archive ACM SIGGRAPH, pp. 1-9, 2008.

[4] Fan Guo, Jin Tang, Zi-Xing Cai, "Image Dehazing Based on Haziness Analysis", International Journal of Automation and Computing 11(1), February 2014, 78-86.

[5] Hongying ZHANG, Qiaolin LIU, Fan YANG, Yadong WU, "Single Image Dehazing Combining Physics Model based and Non-physics Model based Methods", Journal of Computational Information Systems 9: 4 (2013) 16231631.

[6] Y. Y. Schechner, S. G. Narasimhan, and S. K. Nayar, "Instant dehazing of images using polarization", IEEE Computer Society Conference on Computer Vision and Pattern Recognition, pp.325-332, 2001.

[7] Chen Feng , Shaojie Zhuo, Xiaopeng Zhang, Liang Shen, Sabine Susstrunk, "Infra-red Guided Color Image Dehazing", ICIP 2013: 2363

[8] Lex Schaul, Clément Fredembach, and Sabine Susstrunk, "Color Image Dehazing Using The Near-Infrared”, Ecole Polytechnique Fédérale de Lausanne ( EPFL ) School of Computer and Communication Sciences, CH-1015 Lausanne, Switzerland.

[9] Xinwei Liu, Jon Yngve Hardeberg, "Fog Removal Algorithms: survey and perceptual Evaluation", EUVIP, page 118-123. IEEE, (2013).

[10] Schechner, Y and Karpel, N., Clear Underwater Vision, Proceedings of the IEEE CVPR, Vol. 1, 2004, pp. 536-543.

[11] M. D'Zmura, P. Colantoni, K. Knoblauch and B. Laget, "Color transparency", Perception, Volume 26, pp. 471-492, 1997

[12] N. Hautière, J.-P. Tarel, D. Aubert, and E.Dumont, "Blind contrast enhancement assessment by gradient rationing at visible edges", Image Analysis and Stereology Journal, 27(2):87-95, 2008.

[13] John Hagedorn, Micheal D'Zmura, “Color Appearance of Surfaces Viewed Through Fog", Perception, Volume 29, pp. 1169-1184, 2000

[14] F. Metelli, "Additive and substractive color mixture in color transparency", Scientific American 230, pp.90-98, 1974

[15] David L. MacAdam, "Perceptual significance of colorimetric data for colors of plumes and haze", Atmospheric Environment, vol.15, No.10/11, pp. 1797-1803, 1981

[16] S. Fang, J. Zhan, Y. Cao, and R. Rao, 'Improved Single Image Dehazing Using Segmentation", IEEE International Conference on Image Processing (ICIP), 2010, pp. 3589-92.
[17] K. He, J. Sun, X. Tang, "Guided image filtering", Proceedings of the 11th European Conference on Computer Vision, ECCV'10, Part I: 1-14,2010

[18] Tarel, J-P. and Nicolas Hautiere, "Fast visibility restoration from a single color or gray level image", 12th International Conference on Computer Vision, pp. 2201-2208, Year 2009.

[19] Helmholtz H.(1896). Handbuch der Physiologischen Optik: Translated into English by J.P.C Southall in 1924. Translation reprinted in 2000 by Thoemmes Press, 1896, p. 286.

[20] Fritz Ebner, Mark D Fairchild, "Development and testing of a color space (IPT) with improved hue uniformity", Color and Imaging Conference 1998 (1), 8-13, 1998.

[21] Janos Schanda, "Colorimetry: Understanding the CIE System", pp.6465, 2007

[22] C. S. McCamy, H. Marcus, J. G. Davidson, "A Color-Rendition Chart", Journal of Applied Photographic Engineering, Volume 2, Number 3 Summer 1976, pages 95-99

[23] Perre-Jean Lapray, Jean-Baptiste Thomas and Pierre Gouton, " A Multispectral Acquisition System Based On MSFAs”, Color and Imaging Conference, 2014 